\title{
Use of Auxotrophic Mutants to Isolate LL- or DD-Isomers of 2,6-Diaminopimelic Acid
}

\author{
By FARANGIS SALEH AND P. J. WHITE \\ Department of Microbiology, The University, Sheffield SIo $2 T N$
}

(Received I March I976; revised 7 May 1976)

\begin{abstract}
SUMMARY
Pseudomonas aeruginosa PAC7 (a mutant deficient in diaminopimelate epimerase), excreted diaminopimelate (solely LL-isomer) after growth in a minimal medium plus lysine with succinate as carbon source. More diaminopimelate was excreted when bacteria were transferred at the end of the exponential phase of growth into fresh minimal medium without lysine but supplemented with pyruvate and additional $\left(\mathrm{NH}_{4}\right)_{2} \mathrm{SO}_{4}$. The excreted LL-isomer was isolated from the culture filtrate by ionexchange chromatography and purified by crystallization ( $\mathrm{I} \cdot 7 \mathrm{~g} / 9 \mathrm{l}$ culture).

A diaminopimelate-requiring mutant of Bacillus megaterium NCIB758I grew on LL- and/or meso-diaminopimelate but not on the DD-isomer. This mutant was used to isolate the DD-isomer from a mixture of synthetic LL- and DD-diaminopimelate. It was grown in a minimal medium containing glycerol as carbon source and LLplus DD-diaminopimelate at a growth-limiting concentration $\left(300 \mathrm{mg} \mathrm{1}^{-1}\right)$; when growth stopped, the DD-diaminopimelate that remained in the culture was isolated and crystallized ( $\mathrm{r} \circ \mathrm{g} / \mathrm{I}$ I 1 culture).
\end{abstract}

\section{INTRODUCTION}

2,6-Diaminopimelic acid (Dap) exists in three stereoisomeric forms: the LL-, DD- and meso-isomers (Work, 1963). Authentic samples of each are required in studies of the isomeric composition of Dap in walls and spores of bacteria.

Diaminopimelate synthesized chemically is a mixture of all three isomers: about $50 \%$ meso-, $25 \%$ LL- and $25 \%$ DD-isomer (see later). Diaminopimelate can also be isolated from the culture filtrates of lysine-requiring mutants of Escherichia coli (Work, I963); such Dap is a mixture of about $75 \%$ meso- and $25 \%$ LL-isomers (White \& Kelly, 1965). The mesoDap can be separated from the other two isomers by crystallization because it is less soluble in ethanol/water. From the meso- plus LL-Dap mixture obtained by excretion (i.e. 'fermentation Dap'), pure LL-Dap can be recovered after precipitation of meso-Dap. Until recently, commercial Dap was 'fermentation material'; but at present, only synthetic Dap can be bought. Removing the meso-isomer from synthetic Dap leaves a mixture of the LL- and DD-isomers, which are difficult to separate. Work et al. (1955) separated the three isomers by preparing their diamides, treating these with a stereospecific amidase (which deaminated groups in the L-configuration only), and then separating the mixture of free LL-Dap, DD-Dap diamide and meso-Dap (D-)monoamide on an ion-exchange column. Free DD- and meso-Dap were regenerated by acid hydrolysis of the amides. A later modification of this method was described by Wade et al. (1957). These methods are relatively laborious and result in low yields of the pure isomers.

In this paper, we describe the isolation of LL-Dap from cultures of a mutant of Pseudomonas aeruginosa (called PAC7) which excretes solely the LL-isomer (Clarkson \& Meadow, 
1971). The excretion of Dap is small in comparison with that of lysine-requiring mutants of $E$. coli, but all the Dap is the LL-isomer and so there are no losses in resolution steps.

We also describe the use of a Dap-requiring mutant of Bacillus megaterium which can grow in a minimal medium with the LL- and/or meso-isomers (but not DD-Dap) as growth factors. This mutant was grown with a mixture of LL- and DD-Dap, and after it had taken up all the LL-Dap the DD-isomer was recovered from the culture filtrate.

A preliminary report of this work has been given (Saleh \& White, 1975).

\section{METHODS}

Organisms. A mutant derived from Pseudomonas aeruginosa 8602 PACI (NCIBI0848) deficient in diaminopimelate epimerase [now called PAC 7 and described as $l y s-r$ by Clarkson \& Meadow (197I)] was given by Dr Pauline Meadow. The mutant was maintained on slopes of nutrient agar that were incubated overnight at $30^{\circ} \mathrm{C}$ and then stored at $2{ }^{\circ} \mathrm{C}$.

A Dap-requiring mutant of Bacillus megaterium NCIB758I was isolated as described below. It was maintained on slopes of minimal medium FG containing biotin ( $1 \mu \mathrm{g} \mathrm{l}^{-1}$ ) and synthetic Dap (100 $\mathrm{mg} \mathrm{l}^{-1}$ ), that were incubated overnight at $37^{\circ} \mathrm{C}$ and then stored at $2{ }^{\circ} \mathrm{C}$.

Both mutants have been deposited with the National Collection of Industrial Bacteria: their strain numbers are NCIBII250 ( $P$. aeruginosa) and NCIBI I25I (B. megaterium).

Media. All media were prepared with glass-distilled water. The minimal medium for growth of $P$. aeruginosa was that of Clarkson \& Meadow (197I); sodium succinate was the carbon source unless otherwise stated. L-Lysine- $\mathrm{HCl}\left(40\right.$ or $100 \mathrm{mg}^{-1}$ ) was added for growth of the mutant. The basal medium, trace elements and carbon sources were autoclaved separately at $12 \mathrm{I}{ }^{\circ} \mathrm{C}$ for $\mathrm{I} 0 \mathrm{~min}$ and combined after cooling. In tests of the effects of precursors of Dap on the excretion of LL-Dap, supplements were added to the basal medium before autoclaving (except for pyruvate, which was sterilized by filtering).

Minimal medium FG (Fukuda \& Gilvarg, I968) contained (g l-1): $\mathrm{NH}_{4} \mathrm{Cl}, 2 ; \mathrm{Na}_{2} \mathrm{HPO}_{4}$. ${ }_{7} \mathrm{H}_{2} \mathrm{O}, 4.5 ; \mathrm{KH}_{2} \mathrm{PO}_{4}, 3 ; \mathrm{NaCl}, 3 ; \mathrm{MgCl}_{2} .6 \mathrm{H}_{2} \mathrm{O}, 0 . \mathrm{I} ; \mathrm{Na}_{2} \mathrm{SO}_{4}, 0 . \mathrm{II} ; \mathrm{MnCl}_{2} .4 \mathrm{H}_{2} \mathrm{O}$, 0.0I; glucose or glycerol, Io; the pH value was 6.8. Biotin (I $\left.\mu \mathrm{g} \mathrm{l}^{-1}\right)$ and Dap (I00 $\mathrm{mg} \mathrm{l}^{-1}$ ) were added for growth of the mutant. Medium FG with biotin is designated FGB. Solutions of carbon sources were autoclaved separately at I2I ${ }^{\circ} \mathrm{C}$ for Io min and combined with the basal medium after cooling. Solid media were made by adding Difco agar ( $\mathrm{I} \cdot 5 \%$, w/v).

Growth conditions. Organisms were grown on a rotary shaker (New Brunswick Scientific Co., New Brunswick, New Jersey, U.S.A.) at 200 gyrations $/ \mathrm{min}$ at $30^{\circ} \mathrm{C}$ (for $P$. aeruginosa) or at $37^{\circ} \mathrm{C}$ (for $B$. megaterium). The bacterial culture did not occupy more than one-fifth of the volume of the culture flask. Fermenter cultures (I I l) were grown in a New Brunswick Microferm laboratory fermenter with aeration $\left(31 \mathrm{~min}^{-1}\right)$ and mechanical stirring (400 rev.) $\mathrm{min}$ ) at $37^{\circ} \mathrm{C}$. Organisms for inocula were taken from slope cultures; $0.5 \mathrm{ml}$ of a suspension of organisms (about $\mathrm{I} 50 \mu \mathrm{g}$ dry wt for either organism) in sterile water was used per $250 \mathrm{ml}$ of medium.

Measurement of Dap. Dap was measured by a method in which excess lysine does not interfere (Work, 1957) or, when $\mathrm{HCl}$ was present, by the method of Gilvarg (1958).

Assessment of growth. Turbidities of cultures were measured at intervals in $6 \mathrm{~mm}$ diameter tubes using an EEL photoelectric colorimeter with a neutral density filter (no. $\mathrm{I} \cdot 0$ ), and water for the zero setting.

Isolation of diaminopimelic acid from the growth media. The procedure used to isolate LLDap from Pseudomonas cultures and DD-Dap from Bacillus cultures was based on the method of Gilvarg (1959). After appropriate growth (see Results), the bacteria were removed 
by centrifuging (at $23000 \mathrm{~g}$ for $10 \mathrm{~min}$ ); the supernatant liquid was autoclaved for $10 \mathrm{~min}$ at $\mathrm{I} 2 \mathrm{I}{ }^{\circ} \mathrm{C}$ and its $\mathrm{pH}$ was adjusted to 2 with $\mathrm{HCl}$. The liquid was passed at room temperature through a column of Dowex $50\left(\mathrm{H}^{+}\right.$form; $8 \%$ cross-linked; dry mesh 100 to $200 ; 9 \times 12 \mathrm{~cm}$, about $\mathrm{I} 1$ resin bed vol.) at a flow rate of $500 \mathrm{ml} \mathrm{h}^{-1}$. The column was then washed with 21 water, and the Dap was eluted with $2.5 \mathrm{M}-\mathrm{HCl}$ at a rate of $150 \mathrm{ml} \mathrm{h}^{-1}$. After I $1 \mathrm{HCl}$ had emerged, fractions $(200 \mathrm{ml})$ were collected and assayed for Dap (Gilvarg, I958). Fractions 6 to 17 (totalling $2 \cdot 4$ l eluate) contained Dap; these were pooled and dried by rotary evaporation at $42{ }^{\circ} \mathrm{C}$. The oily residue was left overnight with $\mathrm{NaOH}$ pellets in an evacuated desiccator. The resulting solid was resuspended in $20 \mathrm{ml}$ ethanol plus $10 \mathrm{ml}$ redistilled pyridine, kept at $2{ }^{\circ} \mathrm{C}$ for $3 \mathrm{~h}$ with occasional stirring, and then collected on a Buchner filter, washed with pyridine/ethanol ( $\mathrm{I}: 2$, by vol.) and dried over $\mathrm{H}_{2} \mathrm{SO}_{4}$. The dried material was dissolved in $30 \mathrm{ml}$ water by heating, the solution was cooled, and Dap was crystallized by adding ethanol $(30 \mathrm{ml})$. Pure Dap-monohydrochloride (LL or DD) was obtained by a second ethanol/water crystallization.

Isolation of LL- plus DD-Dap from synthetic Dap. The meso-Dap was separated from the LL- and DD-isomers by crystallization from ethanol/water (Hoare \& Work, 1955). The synthetic Dap was dissolved in a minimum amount of boiling water; after cooling, ethanol was added to give a faint permanent turbidity and the solution was left overnight at room temperature. The precipitate was collected and ethanol was again added to the filtrate to give a faint permanent turbidity. The first and second precipitates could be recrystallized from ethanol/water to give pure meso-Dap.

To separate any remaining meso-Dap, the mixture of DD- and LL-Dap left in the filtrate was crystallized as its di-(naphthalene-2-sulphonic acid) salt (Gilvarg, 1959). The aqueous ethanol was evaporated; the solid was redissolved in water to give about I $\mathrm{g} \mathrm{Dap} / \mathrm{Io} \mathrm{ml}$; the solution was acidified with $\mathrm{I} 2 \mathrm{M}-\mathrm{HCl}(2 \mathrm{ml} / \mathrm{IO} \mathrm{ml}$ solution); recrystallized naphthalene-2sulphonic acid was added $(4.2 \mathrm{~g} / \mathrm{IO} \mathrm{ml})$; and the volume was made up with water to $25 \mathrm{ml} /$ g Dap. This solution was boiled, filtered while hot, and left overnight at room temperature. The crystalline precipitate was collected, washed with ethanol and ether, redissolved in $\mathrm{I} \cdot \mathrm{O} \mathrm{M}-\mathrm{HCl}(7.5 \mathrm{ml} / \mathrm{g}$ crude LL- plus DD-Dap) by boiling, and left to stand at room temperature for a few hours. The resulting precipitate was collected and suspended in ethanol $(7.5 \mathrm{ml} /$ $\mathrm{g}$ Dap), and then redistilled pyridine ( $4 \mathrm{ml} / \mathrm{g}$ Dap) was added. The suspension was shaken occasionally during 3 to $4 \mathrm{~h}$ at $2{ }^{\circ} \mathrm{C}$. The solid (LL- plus DD-Dap) was collected and washed with ethanol and ether.

Characterization of isomers of Dap. Methanol/pyridine/water/I0 M- $\mathrm{HCl}(80: 10: 17 \cdot 5: 2 \cdot 5$, by vol.; solvent $\mathrm{A}$; descending) was used for paper chromatography (Whatman no. I, $45 \times 60 \mathrm{~cm}$ ). This solvent separates the LL-isomer of Dap from meso- and DD-Dap, which run together (Rhuland et al., 1955).

Benzyl alcohol/chloroform/methanol/water/15 M-ammonia (30:30:30:6:2, by vol.; solvent B) was used for thin-layer chromatography on plates of Merck silica gel G $(20 \times 20$ $\mathrm{cm} ; 0.25 \mathrm{~mm}$ thick). On thin-layer chromatography in this solvent, the di-DNP (dinitrophenyl) derivative of meso-Dap runs slower than the di-DNP derivatives of LL- or DD-Dap, which run together (Jušić et al., I963).

The isomer composition of Dap was also determined enzymically (Day \& White, 1973). The enzyme diaminopimelate decarboxylase, EC. 4.I.I.20 (White \& Kelly, 1965) is specific for meso-Dap which is quantitatively converted to L-lysine plus $\mathrm{CO}_{2}$. The enzyme diaminopimelate epimerase, EC. 5.I.I.7 (White, Lejeune \& Work, 1969) converts either LL- or mesoDap to an equilibrium mixture, but does not attack DD-Dap. The decarboxylase and pimerase together convert LL-Dap quantitatively to lysine plus $\mathrm{CO}_{2}$. 
Chemicals. 2,6-Diaminopimelic acid was synthesized from 2,6-dibromopimelic acid diethyl ester ( $112 \mathrm{~g}$ batches of ester), as described by Lingens ( 1960 ); the sodium azide used in this preparation was activated as described by Smith (1946). The yield of Dap in several preparations (from the dibromodiester) on this scale was consisiently only $65 \%$, and not $100 \%$ as obtained (on a smaller scale) by Lingens (I960). The dibromodiester was prepared by treating pimelic acid with thionyl chloride and bromine under conditions similar to those used by Ingold (192I) for dibromination of glutaric acid, and then adding the resulting dibromo-di-(acid chloride) to ethanol (Willstätter, I895). The yield of redistilled dibromodiester was $66 \%$ (from pimelic acid).

The synthetic Dap contained $50 \%$ meso-, $25 \%$ LL- and $25 \%$ DD-isomers when assayed by the enzymic methods of Day \& White (1973). Di-DNP derivatives of Dap were prepared as described by Jušić et al. (1963).

\section{RESULTS}

Mutant PAC7 can grow on a minimal medium supplemented only with lysine. Diaminopimelate epimerase is not entirely absent, and the mutant can make enough meso-Dap to support growth when this small amount of meso-Dap is used only for peptidoglycan synthesis, and is not needed to serve as a precursor of lysine as well (Clarkson \& Meadow, I97I). Mutant PAC7 excreted Dap (solely LL-isomer; about $100 \mu \mathrm{g} \mathrm{ml}^{-1}$ ) after growth in minimal medium (carbon source succinate) plus L-lysine $\left(40 \mathrm{mg} \mathrm{l}^{-1}\right)$ at $37^{\circ} \mathrm{C}$ (Clarkson \& Meadow, 197r).

Conditions for greater excretion of Dap were sought. All the trial experiments were done with $25 \mathrm{ml}$ medium in shaken $250 \mathrm{ml}$ flasks. Different carbon sources - glucose, glycerol, trisodium citrate $\left(2 \mathrm{H}_{2} \mathrm{O}\right)$, sodium succinate (all I \%, w/v) - were tested at 25,30 and $37^{\circ} \mathrm{C}$, always with a supplement of L-lysine- $\mathrm{HCl}\left(100 \mathrm{mg} \mathrm{l}^{-1}\right)$. The heaviest growth was obtained using succinate plus lysine at $30^{\circ} \mathrm{C}$, and the Dap in the culture filtrate was measured (Work, I957) every $\mathrm{I}$ or $2 \mathrm{~h}$ during the exponential and stationary phases of growth. Different concentrations of succinate and lysine in the medium were examined (Fig. I). In one series of experiments, lysine- $\mathrm{HCl}$ at $100 \mathrm{mg} \mathrm{l}^{-1}$ gave the greatest Dap excretion; but in another series when a new freeze-dried ampoule of mutant was used, lysine- $\mathrm{HCl}$ at $40 \mathrm{mg} \mathrm{l}^{-1}$ was found to be best. As long as lysine remained in the medium (shown by chromatography with solvent A), there was almost no Dap excretion, but after growth stopped, and lysine became depleted, Dap was rapidly excreted (Fig. 2). Adjusting the $\mathrm{pH}$ value of the culture (in the range 6 to 8) during the period of excretion had no effect on the production of Dap. Since lysine appeared to inhibit Dap excretion, it seemed that more Dap might be excreted if the organisms, when grown, were transferred to fresh medium without lysine. The fresh medium would also give a new supply of carbon and nitrogen for Dap biosynthesis. About $25 \%$ more Dap did accumulate in fresh medium than when the organisms remained in the original growth medium.

Possible precursors of Dap, and additional $\left(\mathrm{NH}_{4}\right)_{2} \mathrm{SO}_{4}$ as nitrogen source, were added to the fresh medium. Excretion of Dap was stimulated between two- and threefold by L-aspartate or L-glutamate or sodium pyruvate (each at $800 \mathrm{mg} \mathrm{l}^{-1}$ ), and various combinations of these were tested. Pyruvate plus $\left(\mathrm{NH}_{4}\right)_{2} \mathrm{SO}_{4}\left(400 \mathrm{mg} \mathrm{l}^{-1}\right)$ was the most effective - in trial experiments up to $400 \mu \mathrm{g} \mathrm{Dap} \mathrm{ml}^{-1}$ was excreted. However, in larger-scale preparations ( $250 \mathrm{ml}$ in 21 flasks), the maximum accumulation was only about $250 \mu \mathrm{g} \mathrm{ml}^{-1}$.

The mutant was grown at $30^{\circ} \mathrm{C}$ with shaking in a total of 91 minimal medium $(250 \mathrm{ml}$ in each 21 flask) containing additional sodium succinate $(1.5 \%, \mathrm{w} / \mathrm{v})$, to increase the yield of bacteria, and L-lysine- $\mathrm{HCl}\left(40 \mathrm{mg} \mathrm{l}^{-1}\right)$. At the end of the exponential phase of growth, bacteria 


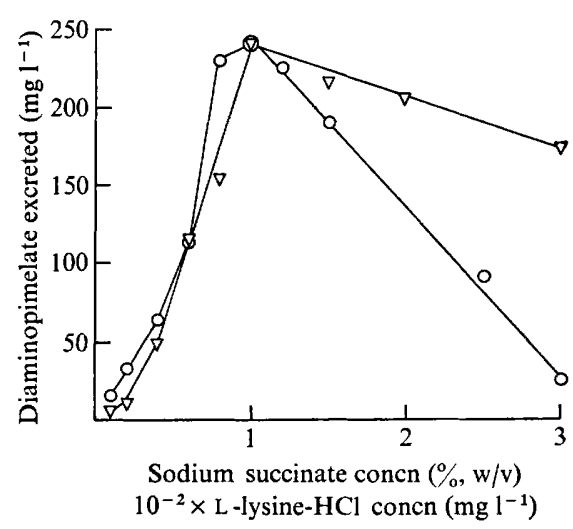

Fig. I

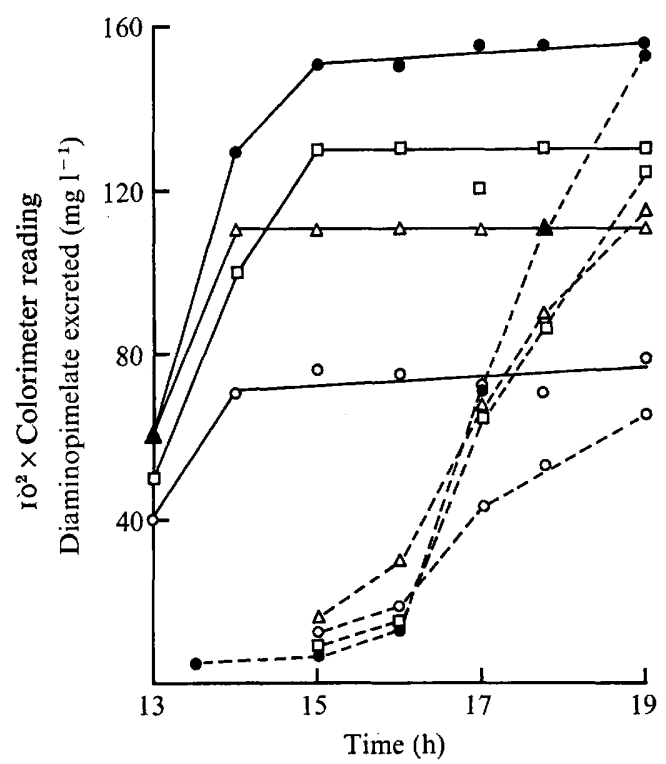

Fig. 2

Fig. I. Excretion of Dap by $P$. aeruginosa PAC7 in media with different concentrations of lysine and succinate. Cultures were incubated at $30^{\circ} \mathrm{C}$ for $40 \mathrm{~h}$ (about $20 \mathrm{~h}$ into the stationary growth phase) before Dap was assayed in the medium. $O$, Dap released into medium containing $\mathrm{I} \%(\mathrm{w} / \mathrm{v})$ sodium succinate, and different concentrations of lysine. $\nabla$, Dap released into medium containing L-lysine- $\mathrm{HCl}\left(100 \mathrm{mg} \mathrm{l}^{-1}\right.$ ) and different concentrations of sodium succinate.

Fig. 2. Growth (-) and release of Dap (- - ) at $30^{\circ} \mathrm{C}$ by $P$. aeruginosa $\mathrm{PAC} 7$ in medium containing $\mathrm{I} \%(\mathrm{w} / \mathrm{v})$ sodium succinate and different concentrations of lysine-HCl $\left(\mathrm{mg} \mathrm{l}^{-1}\right): 0,40 ; \triangle, 60 ; \square$, $80 ; 0,100$.

were transferred into an equal volume of fresh minimal medium containing sodium succinate $(\mathrm{I} \%, \mathrm{w} / \mathrm{v})$, additional $\left(\mathrm{NH}_{4}\right)_{2} \mathrm{SO}_{4}\left(400 \mathrm{mg}^{-1}\right)$ and pyruvate $\left(800 \mathrm{mg} \mathrm{l}^{-1}\right)$, and were reincubated (as before) for $20 \mathrm{~h}$. The LL-Dap was isolated after removing the bacteria (see Methods); the yield, after crystallizing, was $\mathrm{I} \cdot 7 \mathrm{~g}$. It was examined by paper chromatography with solvent A. Ninhydrin ( $0.1 \%$ in acetone) revealed only one spot, in the position expected for LL-Dap: it was green, fading to bright yellow, which is characteristic of Dap. Thinlayer chromatography (of the di-DNP derivative) showed a single yellow spot in the position expected for LL- and DD-Dap. Elementary analysis gave: C, $37 \cdot 20(37 \cdot 09) ; \mathrm{H}, 6 \cdot 84$ $(6.62) ; \mathrm{N}, \mathrm{I} 2.63(\mathrm{I} 2.36) ; \mathrm{Cl}, \mathrm{I} 5.54 \%(15.67 \%)$; values in brackets are those calculated for Dap-monohydrochloride. The optical rotation $[\alpha]_{\mathrm{D}}^{24}$ was $+37.5^{\circ}(c 0.65 \mathrm{~g} / \mathrm{I00} \mathrm{ml} \mathrm{I} \mathrm{M-HCI})$. Work (1963) gave $[\alpha]_{D}^{24}$ for LL-Dap-HCl as $+38.5^{\circ}(c 2.6 \mathrm{~g} / \mathrm{IOO} \mathrm{ml} 5 \mathrm{M}-\mathrm{HCl})$. The Dap was not attacked by diaminopimelate decarboxylase, but was partly converted to meso-Dap by diaminopimelate epimerase. Diaminopimelate decarboxylase plus diaminopimelate epimerase converted all the LL-diaminopimelate to L-lysine plus $\mathrm{CO}_{2}$.

\section{Isolation of a Dap-requiring mutant from B. megaterium $758 \mathrm{I}$}

A Dap-requiring mutant was isolated by using a chemical mutagen, $N$-methyl- $N^{\prime}$-nitro$N$-nitrosoguanidine (Adelberg, Mandel \& Ching Chen, 1965) and penicillin selection (Nester, Schafer \& Lederberg, 1963). Different dilutions of washed organisms after penicillin selection were plated on FGB supplemented with lysine (I00 $\mathrm{mg}^{-1}$ ) plus Dap 


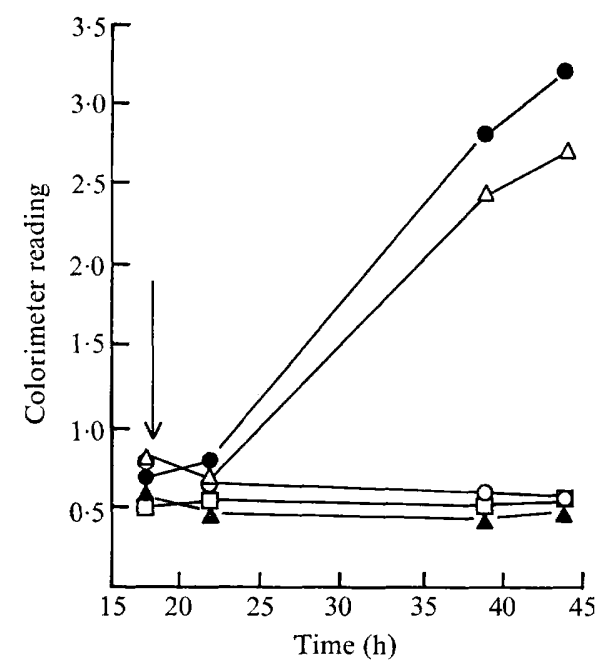

Fig. 3. Growth of $B$. megaterium 758I Dap $^{-}$in FGB medium containing one of the three isomers of Dap or lysine. The Dap mutant was grown at $37^{\circ} \mathrm{C}$ with meso-Dap $\left(20 \mu \mathrm{g} \mathrm{ml}^{-1}\right)$. At the end of the exponential phase of growth, additional $\left(80 \mu \mathrm{g} \mathrm{ml}^{-1}\right)$ meso-Dap (O), LL-Dap $(\triangle)$, DD-Dap (A), or lysine- $\mathrm{HCl}(\square)$ was added at the time indicated by the arrow: $\bigcirc$, no addition.

(50 $\mathrm{mg}^{-1}$ ), and the plates were incubated at $37^{\circ} \mathrm{C}$. The number of survivors was very low. One hundred colonies were tested for Dap-requirement by plating each on different media (FGB alone; FGB plus Dap plus lysine; FGB plus Dap). Two of these colonies required Dap alone, but only one was stable.

\section{Growth of the Dap-requiring mutant with different isomers of Dap}

The nature of the defect in the biosynthesis of Dap in B. megaterium 758 $\mathrm{I} \mathrm{Dap}^{-}$has not yet been established. The mutant grew quickly at $37^{\circ} \mathrm{C}$ in FGB medium supplemented with synthetic Dap (20 to $150 \mathrm{mg} \mathrm{l}^{-1}$ ), but not in FGB alone or FGB plus L-lysine- $\mathrm{HCl}$ (100 mg $\mathrm{1}^{-1}$ ). It also grew in FGB with either LL- or meso-Dap (20 to $150 \mathrm{mg} \mathrm{l}^{-1}$ ) but not with DD-Dap (up to $100 \mathrm{mg}^{-1}$ ) within 4 days. The mutant was grown at $37^{\circ} \mathrm{C}$ with shaking in a series of $250 \mathrm{ml}$ flasks containing FGB medium ( $50 \mathrm{ml})$, meso-Dap $\left(20 \mathrm{mg}^{-1}\right)$, and either glucose or glycerol $(\mathrm{I} \%, \mathrm{w} / \mathrm{v})$ as carbon source. At the end of the exponential phase of growth, LL-, DDor meso-Dap, or L-lysine-HCl (all at $80 \mathrm{mg} \mathrm{l}^{-1}$ ) was added to cultures. Further growth occurred after adding LL- or meso-Dap, but there was no more growth after adding lysine or DD-Dap, or in the control flask with no addition (Fig. 3).

\section{Use of LL-Dap by B. megaterium $758 \mathrm{I}$ Dap-}

The mutant was grown at $37^{\circ} \mathrm{C}$ with shaking in $250 \mathrm{ml}$ flasks containing FGB medium (50 ml) supplemented with LL-Dap (100, 150 or $200 \mathrm{mg}^{-1}$ ), and the Dap remaining in the media was determined after growth ceased. With LL-Dap at up to $150 \mathrm{mg} \mathrm{l}^{-1}$, almost all the Dap was used; but at higher concentrations, some Dap was left. Paper chromatography (solvent A) of $50 \mu \mathrm{l}$ samples from culture filtrates, after growth had stopped, showed a distinct LL-Dap spot from the culture that had initially contained LL-Dap at $200 \mathrm{mg} \mathrm{1}^{-1}$, a very faint LL-Dap spot from the one that had initially contained LL-Dap at $150 \mathrm{mg} \mathrm{l}^{-1}$, and no Dap spot from the culture that had initially contained LL-Dap at $100 \mathrm{mg}^{-1}$. Thus the amount of Dap that the bacteria remove from the medium during growth is limited to 
about $150 \mathrm{mg} \mathrm{l}^{-1}$. Therefore for isolating the DD-isomer it was best to use, as starting material, Dap that already had been enriched with the DD-isomer by preliminary crystallization from synthetic Dap (see Methods).

\section{Use of LL- plus DD-Dap by B. megaterium 7581 Dap}

The mutant was grown as above, but with different concentrations of a mixture of DDand LL-Dap (200, 300 or $400 \mathrm{mg} \mathrm{l}^{-1}$ ); after growth ceased, the concentrations of Dap remaining in the media were $89,14 \mathrm{I}$ and $232 \mathrm{mg} \mathrm{l}^{-1}$, respectively. Paper chromatography of $20 \mu \mathrm{l}$ samples from each medium showed a faint LL-Dap spot from the culture that had initially contained Dap at $400 \mathrm{mg} \mathrm{l}^{-1}$ but no LL-Dap spot was detected in filtrates from the other two cultures.

The mutant was grown in an aerated fermenter at $37^{\circ} \mathrm{C}$ in FGB medium (II l) containing glycerol $(\mathrm{I} \%, \mathrm{w} / \mathrm{v})$ as carbon source, and supplemented with a mixture of LL- and DD-Dap (300 $\left.\mathrm{m} \mathrm{l}^{-1}\right)$. The Dap that remained in the medium after growth ceased was isolated and crystallized (see Methods); the yield of DD-Dap was $\mathrm{I} \cdot 0 \mathrm{~g}$. When the final product was examined by paper chromatography (solvent A), ninhydrin ( $0.1 \%$ in acetone) revealed only one spot, in the position expected for DD-Dap: it was green, fading to yellow. Elementary analysis gave: $\mathrm{C}, 39 \cdot 4(39.8) ; \mathrm{H}, 7.3(6.9)$; $\mathrm{N}, \mathrm{I} 2.9(\mathrm{I} 3.3) ; \mathrm{Cl}, 10.4 \%(10.4 \%)$; values in parentheses are those calculated for a mixture of Dap-monohydrochloride and free Dap (6r $\%$ and $39 \%$ respectively, as suggested by the chloride assay). The optical rotation $[\alpha]_{D}^{24}$ was $-35.9^{\circ}(c 0.65 \mathrm{~g} / \mathrm{I00} \mathrm{ml}$ I $\mathrm{M}-\mathrm{HCl})$. Work (1963) gave $[\alpha]_{\mathrm{D}}^{24}$ for DD-Dap-monohydrochloride as $-38 \cdot 0^{\circ}(c 2 \cdot 6 / 100 \mathrm{ml} 5 \mathrm{M}-\mathrm{HCl})$. The Dap was not attacked by either diaminopimelate decarboxylase, diaminopimelate epimerase, or by the two enzymes together. It did not support growth of B. megaterium 7581 Dap-.

\section{DISCUSSION}

Only about $150 \mathrm{mg}$ LL-Dap (free base) $\mathrm{I}^{-1}$ was isolated from $P$. aeruginosa $\mathrm{PAC} 7$ culture filtrate. Much higher yields of Dap (meso- plus LL-isomers) may be obtained from mutants of $E$. coli that lack diaminopimelate decarboxylase. For example, $2100 \mathrm{mg} \mathrm{1}^{-1}$ is excreted by E. coli 12408 (Work, 1963). From Io $\mathrm{g}$ of such 'fermentation Dap', about I g of pure LL-Dap (and several grammes of meso-isomer) can be isolated by crystallization. Hence, 6 to 71 of 12408 culture should be sufficient to yield $\mathrm{x} \cdot 5 \mathrm{~g}$ LL-Dap, whereas Io 1 of $P$. aeruginosa PAC7 culture is needed to obtain this quantity of LL-Dap (and no meso-isomer is produced). The only advantage of using mutant PAC7 rather than an E. coli mutant is that once the LL-Dap is isolated from the medium, no resolution steps are needed. Lysine synthesis (from succinate) in PAC7 is not completely blocked, and this may be a reason for the relatively low excretion of Dap. Both diaminopimelate epimerase (0.00I unit $/ \mathrm{mg}$ protein) and diaminopimelate decarboxylase $(0.005 \mathrm{unit} / \mathrm{mg}$ protein) were present in an extract of PAC7 (Clarkson \& Meadow, 1971). Lysine causes a feedback inhibition of Dap biosynthesis (see Clarke \& Ornston, 1975), and excretion of Dap by the mutant begins only when lysine is exhausted from the medium. If the mutant continues to form some lysine, biosynthesis of Dap may never become completely derepressed. Therefore, the introduction into PAC7 of a further mutation causing inability to form lysine (as a result of the loss of diaminopimelate decarboxylase), might increase the yield of LL-Dap. However, Clarkson \& Meadow (1971) found that mutants of $P$. aeruginosa 8602 that lacked diaminopimelate decarboxylase had excreted only relatively small amounts of Dap (about $7 \mathrm{mg}^{-1}$ ) by the beginning of the stationary phase of growth. Furthermore, if conversion of meso-Dap to lysine could be 
prevented in PAC7, then the excreted Dap might become a mixture of meso- and LLisomers, which would need to be resolved. It is advantageous for the easy isolation of LL-Dap that diaminopimelate epimerase is not completely blocked in PAC7. If this enzyme were entirely absent, then meso-Dap for peptidoglycan synthesis would have to be provided in the first growth medium, and might possibly mix with the excreted Dap.

Use of the Dap-requiring mutant of $B$. megaterium gives a convenient method of isolating DD-Dap. Although Dap-requiring mutants of $E$. coli also use the LL- and not the DD-isomer, such mutants are less suitable: their rate of uptake of Dap is very low (Leive \& Davis, 1965), and a high concentration of Dap is needed in the medium to support rapid growth (which means that not all the Dap is used when growth ceases); they take up relatively small amounts of Dap (perhaps because their envelopes contain a low proportion of peptidoglycan); and they grow less heavily in defined medium than does B. megaterium. Bacillus megaterium M46 (Fukuda \& Gilvarg, 1968) which requires LL- or meso-Dap plus lysine for growth, is also limited in its ability to remove LL-Dap from the growth medium. In M46, Dap is used only for peptidoglycan synthesis, whereas in B. megaterium 758I Dap $^{-}$both peptidoglycan and lysine derive from Dap.

We wish to thank Dr Pauline Meadow for the culture of $P$. aeruginosa PAC7. Miss Lorna Young carried out the final isolations of DD-Dap. We are grateful to those in the Department of Chemistry, University of Sheffield, who carried out the elementary analyses and measurements of optical rotations.

\section{REFERENCES}

Adelberg, E. A., Mandel, M. \& Ching Chen, G. C. (1965). Optimal conditions for mutagenesis by $N$ methyl- $N^{\prime}$-nitro- $N$-nitrosoguanidine in Escherichia coli $\mathrm{Kr}$. Biochemical and Biophysical Research Communications 18, 788-795.

ClaRKe, P. H. \& ORNSTON, N. (1975). Metabolic pathways and regulation: II. In Genetics and Biochemistry of Pseudomonas, pp. 263-340. Edited by P. H. Clarke and M. H. Richmond. London: Wiley.

Clarkson, C. E. \& Meadow, P. M. (I971). Diaminopimelic acid and lysine auxotrophs of Pseudomonas aeruginosa 8602. Journal of General Microbiology 66, I6I-I69.

DAY, A. \& WHITE, P. J. (1973). Assay of isomers of 2,6-diaminopimelic acid in bacterial walls. Journal of General Microbiology 77, ixx.

Fukuda, A. \& Gilvarg, C. (1968). The relationship of dipicolinate and lysine biosynthesis in Bacillus megaterium. Journal of Biological Chemistry 243, 3871-3876.

Gilvarg, C. (1958). The enzymatic synthesis of diaminopimelic acid. Journal of Biological Chemistry 233 , I $501-1504$.

GILVARG, C. (1959). N-Succinyl-L-diaminopimelic acid. Journal of Biological Chemistry 234, 2955-2959.

HoARE, D. S. \& Work, E. (I955). The stereoisomers of $\alpha, \epsilon$-diaminopimelic acid: their distribution in nature and behaviour towards certain enzyme preparations. Biochemical Journal 6r, 562-568.

INGOLD, C. K. (1921). The conditions underlying the formation of unsaturated and of cyclic compounds from halogenated open-chain derivatives. I. Products derived from $\alpha$-halogenated glutaric acid. Journal of the Chemical Society rig, 305-329.

Jušıć, D., RoY, C., Schocher, A. J. \& WATSON, R. W. (I963). Chromatographic separation of isomeric dinitrophenyl derivatives of alpha-epsilon diaminopimelic acid. Canadian Journal of Biochemistry and Physiology 41, 817-820.

LeIVE, L. \& DAVIS, B D. (1965). The transport of diaminopimelate and cysteine in Escherichia coli. Journal of Biological Chemistry 240, 4362-4369.

LINGENS, F. (1960). Synthese der $\alpha, \epsilon$-Diaminopimelinsäure. Zeitschrift für Naturforschung I5 B, 8I I.

Nester, E. W., Schafer, M. \& Lederberg, M. (1963). Gene linkage in DNA transfer: a cluster of genes concerned with aromatic biosynthesis in Bacillus subtilis. Genetics 48, 529-55I.

Rhuland, L. E., Work, E., Denman, R. F. \& Hoare, D. S. (I955). The behaviour of the isomers of $\alpha, \epsilon-$ diaminopimelic acid on paper chromatograms. Journal of the American Chemical Society 77, 4844-4846.

SALEH, F. \& WhITE, P. J. (1975). Microbial methods for isolating the LL or DD-isomer of 2,6-diaminopimelic acid. Proceedings of the Society for General Microbiology 3, 44-45. 
Smirt, P. A. S. (1946). The Curtius reaction. In Organic Reactions, vol. 3, pp. 337-392. Edited by R. Adams. New York: Wiley.

Wade, R., Birnbaum, S. M., Winitz, M., Koegel, R. J. \& Greenstein, J. P. (1957). Preparation and properties of the isomeric forms of $\alpha$-amino- and $\alpha, \epsilon$-diaminopimelic acid. Journal of the American Chemical Society 79, 648-652.

White, P. J. \& Kelly, B. (1965). Purification and properties of diaminopimelate decarboxylase from Escherichia coli. Biochemical Journal 96, 75-84.

White, P. J., LeJeune, B. \& Work, E. (I969). Assay and properties of diaminopimelate epimerase from Bacillus megaterium. Biochemical Journal Ir3, 589-601.

WILlSTÄTTER, R. (I 895). Zur Kenntniss der Bildung von Kohlenstoffringen. I. Ueberführung von Pimelinsäure in Cyclopentendicarbonsäure. Berichte der Deutschen chemischen Gesellschaft 28, 655-665.

WORK, E. (1957). Reaction of ninhydrin in acid solution with straight chain amino acids containing two amino groups and its application to the estimation of $\alpha, \epsilon$-diaminopimelic acid. Biochemical Journal 67, $416-423$.

Work, E. (1963). $\propto, \epsilon$-Diaminopimelic acid. In Methods in Enzymology, vol. 6, pp. 624-634. Edited by S. P. Colowick and N. O. Kaplan. New York: Academic Press.

Work, E., Birnbaum, S. M., Winitz, M. \& Greenstein, J. P. (1955). Separation of the three isomeric components of synthetic $\alpha, \epsilon$-diaminopimelic acid. Journal of the American Chemical Society 77, 1916I9I8. 